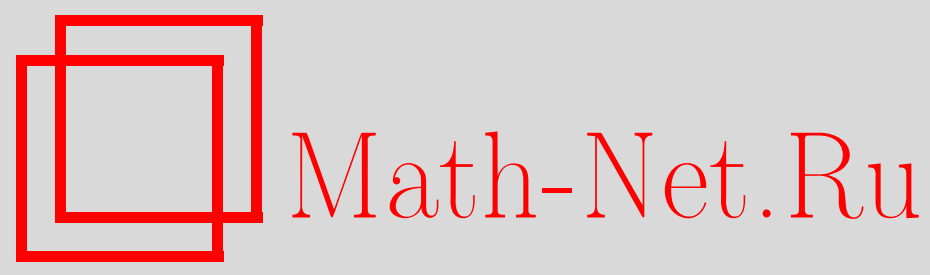

Б. М. Верников, Специальные элементы решетки надкоммутативных многообразий полугрупп, Матем. заметки, 2001, том 70, выпуск 5, 670-678

DOI: https://doi.org/10.4213/mzm779

Использование Общероссийского математического портала Math-Net.Ru подразумевает, что вы прочитали и согласны с пользовательским соглашением http://www.mathnet.ru/rus/agreement

Параметры загрузки:

IP : 54.157 .27 .8

26 апреля 2023 г., $17: 41: 34$

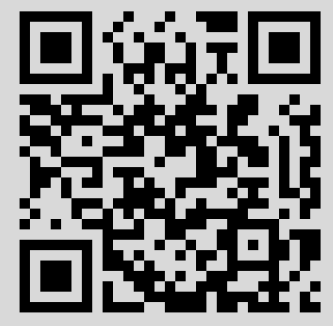




\title{
СПЕЦИАЛЬНЫЕ ЭЛЕМЕНТЫ РЕШЕТКИ НАДКОММУТАТИВНЫХ МНОГООБРАЗИЙ ПОЛУГРУПП
}

\author{
Б. М. Верников
}

\begin{abstract}
Полностью описаны дистрибутивные, кодистрибутивные, стандартные, костандартные и нейтральные элементы решетки надкоммутативных многообразий полугрупп.

Библиографиял: 8 названий.
\end{abstract}

Многообразие полугруп назьвается надкоммутативным, если оно содержит многообразие всех коммутативных полугрупп. Совокупность всех надкоммутативных многообразий полугрупп образует решетку, которую мы будем обозначать через ОС. Строение этой решетки дано в работе М. В. Волкова [1]. В ней показано, что решетка ОС разлагается в подпрямоепроизведение некоторой счетной серии своих интервалов, каждьй из которых антиизоморфен решетке конгруэнций некоторого $G$-множества (напомним, что $G$-множеством называется множество, на котором действует группа, рассматриваемое как унарная алгебра).

В работе [2] нами описаны $G$-множества с различными ограничениями на решетку их конгруэнций (такими, как дистрибутивность, модулярность, полумодулярность, дезарговость и др.). В сочетании с результатами работы [1] это позволило нам в [3] описать надкоммутативные многообразия с теми же ограничениями на решетку их надкоммутативных подмногообразий (см. также развернутый анонс этих результатов [4]). При этом выяснилось, что сама решетка ОС ни одному из рассмотренных ограничений не удовлетворяет (впрочем, это легко вытекает уже из результатов работы [1]). Желание глубже понять свойства решетки ОС как целого естественно приводит к задаче изучения специальных элементов различного рода в решетке ОС. К числу наиболее часто возникаюших в теории решеток типов специальных элементов относятся (ко)дистрибутивные, (ко)стандартные и нейтральные элементы. Напомним, что элемент $x$ решетки $\langle L ; \vee, \wedge\rangle$ назьвается дистрибутивным, если

$$
x \vee(y \wedge z)=(x \vee y) \wedge(x \vee z) \quad \text { для всех } y, z \in L ;
$$

стандартныц, если

$$
(x \vee y) \wedge z=(x \wedge z) \vee(y \wedge z) \quad \text { для всех } y, z \in L
$$

Работа выполнена при поддержке Российского фонда фундаментальных исследований, грант № 97-01-00090, и межвузовской научной программы "Университеты России - фундаментальные исследования" Министерства общего и профессионального образования Российской Федерации, проект № 617 . 
нейтральным, если

$$
(x \wedge y) \vee(y \wedge z) \vee(z \wedge x)=(x \vee y) \wedge(y \vee z) \wedge(z \vee x) \quad \text { для всех } y, z \in L
$$

Кодистрибутивные и костандартные элементы определяются двойственно к дистрибутивньм и стандартньм соответственно. Обширную информацию об элементах всех указанных типов, показывающую естественность и важность их изучения, можно найти, например, в $§$ III.2 монографии [5]. Кратко говоря, дистрибутивные и кодистрибутивные элементы “отвечают" за гомоморфизмы решетки на свои интервалы, а нейтральные - за ее разложение в подпрямое произведение своих интервалов. Отметим еще, что всякий (ко)стандартньй элемент (ко)дистрибутивен и что элемент нейтрален тогда и только тогда, ког да он стандартен и костандартен одновременно (см., например, теорему III.2.5 в [5]). С другой стороны, (ко)дистрибутивньй элемент не обязан быть (ко)стандартным, а (ко)стандартньй - нейтральным.

Работа состоит из двух пунктов. В п. 1 изучаются (ко)дистрибутивные, (ко)стандартные и нейтральные элементы в решетках конгруэнций $G$-множеств. Доказьваемая в этом пункте теорема 1 дает описание таких элементов для $G$-множеств, удовлетворяющих некоторому дополнительному условию. Основньм результатом работы является доказьваемая в п. 2 теорема 2. Она дает полное описание элементов пяти указанных типов в решетке ОС. Доказательство теоремы 2 опирается на результаты работы [1] и теорему 1.

Автор благодарит профессора М. В.Волкова за полезные обсуждения и рецензента за указание на неточность в первоначальном варианте рукописи и некоторые другие конструктивные замечания.

1. $G$-множества. Выше мы уже напомнили понятие $G$-множества. Более формально оно вводится следующим образом. Пусть $A$ - непустое множество, $G$ - группа, а $\varphi$ - гомоморфизм из $G$ в группу всех перестановок множества $A$. Каждому элементу $g \in G$ поставим в соответствие унарную операцию $g^{*}$ на $A$, задаваемую правилом $g^{*}(a)=(\varphi(g))(a)$ для всякого $a \in A$. Алгебра с носителем $A$ и множеством операций $\left\{g^{*} \mid g \in G\right\}$ и назьвается $G$-множсеством. Некоторая первоначальная информация о $G$-множествах и, в частности, о их конгруэнциях имеется, например, в монографии [6].

Приведем ряд необходимых определений и обозначений. $G$-множество $A$ называется транзитивным, если для любых двух элементов $x, y \in A$ существует $g \in G$ такой, что $g^{*}(x)=y$. Транзитивное $G$-подмножество $G$-множества $A$ назьвается орбитой в $A$. Ясно, что любое $G$-множество является дизъюнктньм объединением всех своих орбит. Множество всех орбит $G$-множества $A$ обозначим через $\operatorname{Orb}(A)$. Как обычно, решетку конгруэнций на $A$ будем обозначать через $\operatorname{Con}(A)$, а решетку эквивалентностей на множестве $X$ - через $\operatorname{Eq}(X)$. Пусть $\alpha \in \operatorname{Con}(A)$, а $B$ и $C$ - различные орбиты в $A$. Будем говорить, что

$\alpha$ изолирует орбиту $B$, если из того, что $x \in B$ и $x \alpha y$, вытекает, что $y \in B$;

$\alpha$ разделяет орбиты $B u C$, если несуществует элементов $x \in B$ и $y \in C$ таких, что $x \alpha y$;

$\alpha$ связывает орбиты $B$ и $C$, если $\alpha$ не разделяет их, т.е. если сушествуют элементы $x \in B$ и $y \in C$ такие, что $x \alpha y$;

$\alpha$ склеивает орбиты $B u C$, если $x \alpha y$ для любых элементов $x, y \in B \cup C$.

В дальнейшем нам понадобится следующая простая 
Лемма 1. Если конгруэниия $\alpha$ на $G$-множестве $A$ связывает его различные орбиты $B$ и $C$, то для всякого әлемента $b \in B$ существует әлемент $c \in C$ такой, что bаc.

ДокАЗАТЕЛЬСтво. По условию существуют элементы $x \in B$ и $y \in C$ такие, что $x \alpha y$. Пусть теперь $b \in B$. В силу транзитивности $B$ сушествует элемент $g \in G$ такой, что $b=g^{*}(x)$. Положим $c=g^{*}(y)$. Тогда $c \in C$ и $b \alpha c$. Лемма доказана.

Конгруэнцию $\alpha$ будем называть жадной, если она склеивает любые две орбиты, которые она связывает. Множество всех жадных конгруэнций $G$-множества $A$ обозначим через $\mathrm{GCon}(A)$. В работе [2] показано, что $\mathrm{GCon}(A)$ - подрешетка в $\operatorname{Con}(A)$, и изучено строение этой подрешетки. Сформулируем эти результаты в удобном для дальнейшего виде. Для всякой конгруэнции $\alpha$ на $A$ определим бинарное отношение $\alpha^{*}$ на $\operatorname{Orb}(A)$ правилом: если $B, C \in \operatorname{Orb}(A)$, то $B \alpha^{*} C$ тогда и только тогда, когда либо $B=C$, либо $\alpha$ связывает $B$ и $C$. Очевидно, что $\alpha^{*}$ - отношение эквивалентности на $\operatorname{Orb}(A)$.

ЛЕмма 2 [2, лемма 1.1 и предложение 1.2]. Пусть $A-G$-множсество $u \operatorname{Orb}(A)=$ $\left\{A_{i} \mid i \in I\right\}$. Мнохсество $\mathrm{GCon}(A)$ является подрешеткой решетки $\operatorname{Con}(A)$. Отображение $f$ из $\mathrm{GCon}(A)$ в $\operatorname{Eq}(\operatorname{Orb}(A)) \times \prod_{i \in I} \operatorname{Con}\left(A_{i}\right)$, задаваемое правилом

$$
f(\alpha)=\left(\alpha^{*} ; \ldots, \alpha_{i}, \ldots\right),
$$

әде $\alpha_{i}$ есть ограничение конгруэниии $\alpha \in \operatorname{GCon}(A)$ на орбиту $A_{i}$, является изоморфным вложением.

Пусть $A-G$-множество и $a \in A$. Положим

$$
\operatorname{Stab}_{A}(a)=\left\{g \in G \mid g^{*}(a)=a\right\} .
$$

Ясно, что $\operatorname{Stab}_{A}(a)$ - подгруппа в $G$. Она называется стабилизатором әлемента $a$ B $A$.

Пусть $B$ и $C$ - различные орбиты $G$-множества $A, b \in B$ и $c \in C$. Обозначим через $\rho_{b, c}$ бинарное отношение на $A$, заданное правилом: $x \rho_{b, c} y$ тогда и только тогда, когда либо $x=y$, либо существует $g \in G$ такой, что $\{x, y\}=\left\{g^{*}(b), g^{*}(c)\right\}$.

Лемма 3. Если $\operatorname{Stab}_{A}(b)=\operatorname{Stab}_{A}(c)$, то $\rho_{b, c}-$ конгруәниия на $A$.

ДокАЗАтЕЛЬСтво. Рефлексивность, симметричность и стабильность отношения $\rho_{b, c}$ очевидны. Осталось убедиться в его транзитивности. Пусть $x \rho_{b, c} y \rho_{b, c} z$. Разумеется, можно считать, что $x \neq y$ и $y \neq z$. Поэтому без ограничения общности можно считать, что сушествуют элементы $g_{1}, g_{2} \in G$ такие, что $x=g_{1}^{*}(b), y=g_{1}^{*}(c)$ и либо $y=g_{2}^{*}(c), z=g_{2}^{*}(b)$, либо $y=g_{2}^{*}(b), z=g_{2}^{*}(c)$. Однако случай, когда $y=g_{2}^{*}(b)$, невозможен, поскольку $y=g_{1}^{*}(c) \in C$, а $g_{2}^{*}(b) \in B$. Итак, $y=g_{1}^{*}(c)=g_{2}^{*}(c)$. Следовательно, $g_{2}^{-1} g_{1} \in \operatorname{Stab}_{A}(c)=\operatorname{Stab}_{A}(b)$. Следовательно, $x=g_{1}^{*}(b)=g_{2}^{*}(b)=z$. В частности, $x \rho_{b, c} z$. Лемма доказана.

Пусть $\mu$-конгруэнция $G$-множества $A$, которая связывает, но не склеивает некоторые его различные орбиты $B$ и $C$. Ясно, что ограничение $\mu$ на $B$ не является универсальным отношением. Следовательно, $(v, w) \notin \mu$ для некоторых элементов $v, w \in B$. В силу леммы 1 сушествует элемент $w^{\prime} \in C$ такой, что $w \mu w^{\prime}$.

Лемма 4. Если $\operatorname{Stab}_{A}(v)=\operatorname{Stab}_{A}\left(w^{\prime}\right)$, то конгруэниия $\mu \wedge \rho_{v, w^{\prime}}$ разделяет орбumbl $B u C$. 
ДокАЗАТЕЛЬСТво. Прежде всего, отметим, что в силу леммы $3 \rho_{v, w^{\prime}}-$ конгруэнция на $A$. Предположим, что $(x, y) \in \mu \wedge \rho_{v, w^{\prime}}$ для некоторых элементов $x \in B$ и $y \in C$. Ясно, что $x \neq y$. Поскольку $(x, y) \in \rho_{v, w^{\prime}}$, существует $g \in G$ такой, что $x=g^{*}(v)$ и $y=g^{*}\left(w^{\prime}\right)$. Учитьвая, что $(x, y) \in \mu$, получаем, что $v \mu w^{\prime}$. Но тогда $v \mu w$, что противоречит выбору элементов $v$ и $w$. Лемма доказана.

Если $\mu$ - конгруэнция $G$-множества $A$, а $X$ - орбита в $A$, то мы будем обозначать ограничение $\mu$ на $X$ через $\mu_{X}$.

Основным результатом данного пункта является

Tеорема 1. Пусть $A$ - нетранзитивное $G$-множество такое, что $\operatorname{Stab}_{A}(x)=$ $\operatorname{Stab}_{A}(y)$ для любых әлементов $x, y \in A$. Для произвольной конгруәниии $\alpha$ на $A$ следуюшие условия әквивалентны:

а) $\alpha$ - дистрибутивный әлемент решетки $\operatorname{Con}(A)$;

б) $\alpha$-кодистрибутивный әлемент решетки $\operatorname{Con}(A)$;

в) $\alpha$ - стандартный әлемент решетки $\operatorname{Con}(A)$;

г) $\alpha$ - костандартный элемент решетки $\operatorname{Con}(A)$;

д) $\alpha$ - нейтральныи әлемент решетки $\operatorname{Con}(A)$;

е) $\alpha$ - либо универсальное отношение, либо отношение равенства.

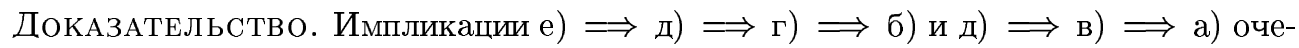
видны. Поэтому в доказательстве нуждаются лишь импликации а) $\Longrightarrow$ е) и б) $\Longrightarrow$ е). Итак, пусть $\alpha$-конгруэнция на $A$, отличная от универсального отношения и являющаяся дистрибутивным или кодистрибутивным элементом в $\operatorname{Con}(A)$. Надо доказать, что $\alpha$ - отношение равенства.

Проверим сначала, что выполнены следующие утверждения:

А) $\alpha$ изолирует каждую орбиту $G$-множества $A$;

Б) если конгруэнция $\beta$ на $A$ связывает некоторую орбиту $B$ с некоторой другой орбитой, то ограничение $\alpha$ на $B$ содержится в ограничении $\beta$ на $B$.

Эта проверка естественно распадается на два случая.

Случай 1. $\alpha$ - дистрибутивньй элемент в $\operatorname{Con}(A)$.

Прежде всего, докажем, что $\alpha$ - жадная конгруэнция. Предположим, что это не так. Тогда сушествуют различные орбиты $B$ и $C$ в $A$, которые связываются, но не склеиваются конгруэнцией $\alpha$. Определим конгруэнции $\beta$ и $\gamma$ на $A$ следуюшим образом: $v \beta w$ тогда и только тогда, когда либо $v=w$, либо $v, w \in B$ и $v \alpha w$, либо $v, w \in C$; $v \gamma w$ тогда и только тогда, когда либо $v=w$, либо $v, w \in B$, либо $v, w \in C$ и $v \alpha w$. Пусть $x, y \in B$ и $(x, y) \notin \alpha$ (такие элементы существуют, поскольку $\alpha$ не склеивает $B$ и $C)$. Ясно, что $(x, y) \in \gamma \subseteq \alpha \vee \gamma$. В силу леммы 1 существуют элементы $x^{\prime}, y^{\prime} \in C$ такие, что $x \alpha x^{\prime}$ и $y \alpha y^{\prime}$. Тогда $x \alpha x^{\prime} \beta y^{\prime} \alpha y$, и потому $(x, y) \in \alpha \vee \beta$. Следовательно, $(x, y) \in(\alpha \vee \beta) \wedge(\alpha \vee \gamma)$. Предположим теперь, что $(x, y) \in \alpha \vee(\beta \wedge \gamma)$. Тогда существует последовательность $x_{0}, x_{1}, \ldots, x_{n}$ элементов из $A$ такая, что $x_{0}=x$, $x_{n}=y$ и для всякого $i=0,1, \ldots, n-1$ либо $x_{i} \alpha x_{i+1}$, либо $\left(x_{i}, x_{i+1}\right) \in \beta \wedge \gamma$. Пусть $x_{0}, x_{1}, \ldots, x_{n}-$ кратчайшая последовательность с таким свойством. Предположим, что $x_{0}, x_{1}, \ldots, x_{i-1} \in B \cup C$, но $x_{i} \notin B \cup C$ для некоторого $i$. Ясно, что $0<i<n$. Из определения конгруэнций $\beta$ и $\gamma$ вытекает, что $x_{i-1} \alpha x_{i} \alpha x_{i+1}$. Но это противоречит тому, что $x_{0}, x_{1}, \ldots, x_{n}-$ кратчайшая последовательность с указанным вьше свойством. Итак, $x_{0}, x_{1}, \ldots, x_{n} \in B \cup C$. Предположим теперь, что $x_{0}, x_{1}, \ldots, x_{i-1} \in B$, но $x_{i} \in C$ 
для некоторого $i$. Ясно, что вновь $0<i<n$. Поскольку конгруэнции $\beta$ и $\gamma$ разделяют орбиты $B$ и $C$, получаем, что $x_{i-1} \alpha x_{i}$. Если $x_{i+1} \in B$, то по той же причине $x_{i} \alpha x_{i+1}$. Если же $x_{i+1} \in C$, то вновь $x_{i} \alpha x_{i+1}$, поскольку ограничение конгруэнции $\beta \wedge \gamma$ на $C$ совпадает с ограничением $\alpha$ на $C$. Итак, в любом случае $x_{i-1} \alpha x_{i} \alpha x_{i+1}$. Это вновь противоречит тому, что $x_{0}, x_{1}, \ldots, x_{n}$ - кратчайшая последовательность с указанным вьше свойством. Таким образом, $x_{0}, x_{1}, \ldots, x_{n} \in B$. Учитывая, что ограничение конгруэнции $\beta \wedge \gamma$ на $B$ совпадает с ограничением $\alpha$ на $B$, получаем, что $x=x_{0} \alpha x_{n}=y$, что противоречит выбору элементов $x$ и $y$. Мы показали, что $\alpha \vee(\beta \wedge \gamma) \neq(\alpha \vee \beta) \wedge(\alpha \vee \gamma)$ вопреки предположению о том, что $\alpha$ - дистрибутивный элемент решетки Con $(A)$. Следовательно, $\alpha-$ жадная конгруэнция.

Из леммы 2 теперь непосредственно вытекает, что эквивалентность $\alpha^{*}-$ дистрибутивньй элемент решетки $\mathrm{Eq}(\mathrm{Orb}(A))$. Хорошо известно и легко проверяется, что отношение эквивалентности на произвольном множестве не содержит ни дистрибутивных, ни кодистрибутивных элементов, отличных от универсального отношения и отношения равенства. Ясно, что $\alpha^{*}$ не является универсальным отношением на $\operatorname{Orb}(A)$, так как в противном случае из жадности $\alpha$ вытекало бы, что $\alpha$ - универсальное отношение на $A$. Следовательно, $\alpha^{*}$ - отношение равенства на $\operatorname{Orb}(A)$. Это означает, что вьполнено утверждение А).

Осталось проверить утверждение Б). Пусть $\beta$-конгруэнция на $A$, которая связывает некоторую орбиту $B G$-множества $A$ с некоторой другой его орбитой $C$. Предположим, что $\alpha_{B} \nsubseteq \beta_{B}$. Тогда существуют элементы $x, y \in B$ такие, что $x \alpha y$, но $(x, y) \notin \beta$. В силу леммы 1 существует элемент $y^{\prime} \in C$ такой, что $y \beta y^{\prime}$. Положим $\gamma=\rho_{x, y^{\prime}}$. По условию $\operatorname{Stab}_{A}(x)=\operatorname{Stab}_{A}\left(y^{\prime}\right)$. В силу леммы $3 \gamma-$ конгруэнция на $A$, а в силу леммы 4 конгруэнция $\beta \wedge \gamma$ разделяет орбиты $B$ и $C$. Поскольку $\alpha$ изолирует каждую орбиту, конгруэнция $\alpha \vee(\beta \wedge \gamma)$ также разделяет орбиты $B$ и $C$. В частности, $\left(x, y^{\prime}\right) \notin \alpha \vee(\beta \wedge \gamma)$. В то же время $\left(x, y^{\prime}\right) \in \alpha \vee \beta$, поскольку $x \alpha y \beta y^{\prime}$, и $x \gamma y^{\prime}$ по построению $\gamma$. Следовательно, $\left(x, y^{\prime}\right) \in(\alpha \vee \beta) \wedge \gamma \subseteq(\alpha \vee \beta) \wedge(\alpha \vee \gamma)$. Таким образом, $\alpha \vee(\beta \wedge \gamma) \neq(\alpha \vee \beta) \wedge(\alpha \vee \gamma)$ вопреки предположению о том, что $\alpha$ - дистрибутивньй элемент решетки $\operatorname{Con}(A)$. Это противоречие доказьвает, что $\alpha_{B} \subseteq \beta_{B}$, что и требовалось.

Случай 2. $\alpha$ - кодистрибутивньй элемент в $\operatorname{Con}(A)$.

Как и при рассмотрении случая 1 , проверим сначала, что $\alpha$ - жадная конгруэнция. Предположим противное. Тогда существуют различные орбиты $B$ и $C$ в $A$, которые связываются, но не склеиваются конгруэнцией $\alpha$. Определим конгруэнцию $\beta$ на $A$ следующим образом: $v \beta w$ тогда и только тогда, когда либо $v=w$, либо $v, w \in B$ и $v \alpha w$, либо $v, w \in C$. Пусть $x, y \in B$ и $(x, y) \notin \alpha$ (такие элементы существуют, поскольку $\alpha$ не склеивает $B$ и $C$ ). В силу леммы 1 существуют элементы $x^{\prime}, y^{\prime} \in C$ такие, что $x \alpha x^{\prime}$ и $y \alpha y^{\prime}$. Положим $\gamma=\rho_{x, y^{\prime}}$. Как и в случае 1 , из леммы 3 и условия теоремы вытекает, что $\gamma$ - конгруэнция на $A$. Далее, $x \gamma y^{\prime} \beta x^{\prime}$, откуда $\left(x, x^{\prime}\right) \in \beta \vee \gamma$. Поскольку, кроме того, $x \alpha x^{\prime}$, получаем, что $\left(x, x^{\prime}\right) \in \alpha \wedge(\beta \vee \gamma)$. С другой стороны, из определения конгруэнции $\beta$ и леммы 4 вытекает, что каждая из конгруэнций $\alpha \wedge \beta$ и $\alpha \wedge \gamma$ разделяет орбиты $B$ и $C$. Учитьвая, что $x \in B$, а $x^{\prime} \in C$, получаем, что $\left(x, x^{\prime}\right) \notin(\alpha \wedge \beta) \vee(\alpha \wedge \gamma)$. Следовательно, $\alpha \wedge(\beta \vee \gamma) \neq(\alpha \wedge \beta) \vee(\alpha \wedge \gamma)$ вопреки предположению о том, что $\alpha-$ кодистрибутивный элемент в $\operatorname{Con}(A)$. Следовательно, $\alpha-$ жадная конгруэнция. Как и в случае 1 , отсюда вытекает, что выполнено утверждение А).

Остается проверить вьполнение утверждения Б). Пусть $\beta$ - конгруэнция на $A$, которая связьвает некоторую орбиту $B G$-множества $A$ с некоторой его другой орбитой $C$. Предположим, что $\alpha_{B} \nsubseteq \beta_{B}$. Тогда существуют элементы $x, y \in B$ такие, что $x \alpha y$, но $(x, y) \notin \beta$. Определим конгруэнцию $\gamma$ на $A$ правилом: $v \gamma w$ тогда и только тогда, когда 
либо $v=w$, либо $v, w \in C$. В силу леммы 1 существуют элементы $x^{\prime}, y^{\prime} \in C$ такие, что $x \beta x^{\prime}$ и $y \beta y^{\prime}$. Тогда $x \beta x^{\prime} \gamma y^{\prime} \beta y$ и потому $(x, y) \in \beta \vee \gamma$. Поскольку $x \alpha y$, получаем, что $(x, y) \in \alpha \wedge(\beta \vee \gamma)$. Предположим, что $(x, y) \in(\alpha \wedge \beta) \vee(\alpha \wedge \gamma)$. Тогда существует последовательность $x_{0}, x_{1}, \ldots, x_{n}$ элементов из $A$ такая, что $x_{0}=x, x_{n}=y$ и для всякого $i=0,1, \ldots, n-1$ либо $\left(x_{i}, x_{i+1}\right) \in \alpha \wedge \beta$, либо $\left(x_{i}, x_{i+1}\right) \in \alpha \wedge \gamma$. В силу утверждения A) каждая из конгруэнций $\alpha \wedge \beta$ и $\alpha \wedge \gamma$ изолирует $B$. Поскольку $x_{0}=x \in B$, отсюда вытекает, что $x_{1}, \ldots, x_{n} \in B$. Учитывая, что ограничение конгруэнции $\alpha \wedge \gamma$ на $B$ есть отношение равенства, получаем, что $(x, y) \in \alpha \wedge \beta$. Но это противоречит тому, что $(x, y) \notin \beta$. Итак, $(x, y) \notin(\alpha \wedge \beta) \vee(\alpha \wedge \gamma)$. Следовательно, $\alpha \wedge(\beta \vee \gamma) \neq(\alpha \wedge \beta) \vee(\alpha \wedge \gamma)$ вопреки предположению о том, что $\alpha$ - кодистрибутивный элемент в $\operatorname{Con}(A)$. Следовательно, $\alpha_{B} \subseteq \beta_{B}$, что и требовалось доказать.

Итак, утверждения А) и Б) выполнены в любом случае. Проверим, что ограничение $\alpha$ на произвольную орбиту является отношением равенства. Этого достаточно для завершения доказательства теоремы, так как в этом случае из утверждения А) вытекает, что $\alpha$-отношение равенства.

Пусть $B$ и $C$ - различные орбиты в $A$. Зафиксируем элементы $b_{0} \in B$ и $c_{0} \in C$. По

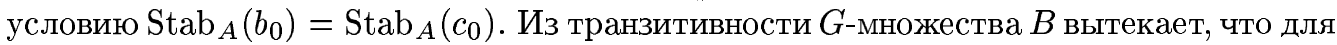
всякого $b \in B$ существует элемент $g_{b} \in G$ такой, что $b=g_{b}^{*}\left(b_{0}\right)$. Определим отображение $\varphi$ из $B$ в $C$ правилом: если $b \in B$, то $\varphi(b)=g_{b}^{*}\left(c_{0}\right)$. Ясно, что $\varphi-$ сюръективный гомоморфизм из $B$ в $C$. Проверим, что $\varphi$ взаимно однозначно. Предположим, что $\varphi(b)=\varphi(d)$ для некоторых элементов $b, d \in B$. Иными словами, $g_{b}^{*}\left(c_{0}\right)=g_{d}^{*}\left(c_{0}\right)$. Тогда $g_{d}^{-1} g_{b} \in \operatorname{Stab}_{A}\left(c_{0}\right)=\operatorname{Stab}_{A}\left(b_{0}\right)$. Следовательно, $d=g_{d}^{*}\left(b_{0}\right)=g_{b}^{*}\left(b_{0}\right)=b$. Итак, $\varphi-$ изоморфизм из $B$ на $C$.

Определим бинарное отношение $\beta$ на $A$ правилом: $x \beta y$ тогда и только тогда, когда либо $x=y$, либо $x \in B, y \in C$ и $y=\varphi(x)$, либо $x \in C, y \in B$ и $x=\varphi(y)$. Из того, что $\varphi$ - изоморфизм, легко вытекает, что $\beta$ - конгруэнция на $A$. Ясно, что она связьвает орбиты $B$ и $C$. В силу утверждения Б) $\alpha_{B} \subseteq \beta_{B}$. Поскольку $\beta_{B}$ - отношение равенства, получаем, что тем же свойством обладает и $\alpha_{B}$. Итак, ограничение $\alpha$ на произвольную неодноэлементную орбиту является отношением равенства.

Теорема доказана.

Основное ограничение, наложенное на $G$-множество в теореме 1 (равенство стабилизаторов любых двух элементов), является достаточно сильным. Но в рамках данной работы его можно считать естественньп, поскольку, как мы увидим в п. 2, оно выполнено для всех $G$-множеств, возникающих при описании строения решетки надкоммутативных многообразий полугрупп.

2. Надкоммутативные многообразия. Мы начнем этот пункт с воспроизведения необходимых нам результатов работы [1].

Пусть $F$ - абсолютно свободная полугруппа над счетньм алфавитом $\left\{x_{1}, x_{2}, \ldots\right.$, $\left.x_{n}, \ldots\right\}$. Если $u \in F$, то множество всех букв, входящих в запись $u$, будем обозначать через $c(u)$, длину слова $u$ - через $\ell(u)$, а число вхождений буквы $x_{i}$ в запись $u$ - через $\ell_{i}(u)$. Если $u \in F$ и $c(u)=\left\{x_{1}, x_{2}, \ldots, x_{m}\right\}$, то через $\operatorname{part}(u)$ мы обозначаем последовательность $\left(\ell_{1}(u), \ell_{2}(u), \ldots, \ell_{m}(u)\right)$. Символом 三 обозначается отношение равенства на $F$.

Напомним, что тождество $u=v$ назьвается уравновешенным, если $\ell_{i}(u)=\ell_{i}(v)$ для всякого $i$. Хорошо известно, что многообразие полугрупп надкоммутативно тогда и только тогда, когда оно задается только уравновешенньми тождествами. Пусть 
$u=v$ - уравновешенное тождество. Тогда, очевидно, $c(u)=c(v), \ell(u)=\ell(v)$ и $\operatorname{part}(u)=\operatorname{part}(v)$. Второе из этих равенств позволяет определить длину уравновешенного тождества $u=v$ как длину каждого из слов $u$ и $v$.

Пусть $n$ - натуральное число и $n \geqslant 2$. Обозначим через $\mathscr{C}_{n}$ многообразие полугрупा, заданное всеми уравновешенными тождествами длины $\geqslant n$. Ясно, что

$$
\mathscr{C} \mathscr{O} \mathscr{M}=\mathscr{C}_{2} \subset \mathscr{C}_{3} \subset \cdots \subset \mathscr{C}_{n} \subset \cdots \subset \mathscr{S} \mathscr{E} \mathscr{M}
$$

где $\mathscr{C} \mathscr{O} \mathscr{M}$ - многообразие всех коммутативных полугрупп, а $\mathscr{S} \mathscr{E} \mathscr{M}$ - многообразие всех полугрупп. Пусть, далее, $m$ - натуральное число такое, что $2 \leqslant m \leqslant n$. Обозначим через $\mathscr{C}_{n, m}$ многообразие полугруп, заданное всеми уравновешенными тождествами длины $>n$ и всеми уравновешенными тождествами длины $n$, зависящими от $\leqslant m$ букв. Для удобства обозначений положим также $\mathscr{C}_{n, 1}=\mathscr{C}_{n+1}$. Ясно, что

$$
\mathscr{C}_{n}=\mathscr{C}_{n, n} \subset \mathscr{C}_{n, n-1} \subset \cdots \subset \mathscr{C}_{n, 2} \subset \mathscr{C}_{n, 1}=\mathscr{C}_{n+1}
$$

Пусть, наконец, $\lambda=\left(\lambda_{1}, \lambda_{2}, \ldots, \lambda_{m}\right)$ - разбиение числа $n$ на $m$ частей, т.е. последовательность $\left(\lambda_{1}, \lambda_{2}, \ldots, \lambda_{m}\right)$ натуральных чисел такая, что

$$
\lambda_{1} \geqslant \lambda_{2} \geqslant \cdots \geqslant \lambda_{m} \quad \text { и } \quad \sum_{i=1}^{m} \lambda_{i}=n .
$$

Обозначим через $\mathscr{C}_{\lambda}$ подмногообразие многообразия $\mathscr{C}_{n, m-1}$, заданное внутри последнего всеми уравновешенными тождествами $u=v$ длины $n$ такими, что $c(u)=\left\{x_{1}, x_{2}\right.$, $\left.\ldots, x_{m}\right\}$ и $\operatorname{part}(u)=\lambda$. Ясно, что $\mathscr{C}_{n, m} \subset \mathscr{C}_{\lambda} \subset \mathscr{C}_{n, m-1}$. Интервал $\left[\mathscr{C}_{\lambda}, \mathscr{C}_{n, m-1}\right]$ решетки ОС обозначим через $I_{\lambda}$.

Обозначим через $\Lambda$ множество всех невозрастающих последовательностей натуральных чисел длины $\geqslant 2$. Ясно, что если $\lambda$ - разбиение числа $n$ на $m$ частей, то $\lambda \in \Lambda$, и наоборот, если $\lambda=\left(\lambda_{1}, \lambda_{2}, \ldots, \lambda_{m}\right) \in \Lambda$, то $\lambda$ - разбиение числа $n=\sum_{i=1}^{m} \lambda_{i}$ на $m$ частей.

Лемма 5 [1, предложения 2.2 и 3.1]. Решетка всех надкоммутативных многообразий полугрупп разлагается в подпрямое произведение интервалов вида $I_{\lambda}$, где $\lambda$ пробегает множсество $\Lambda$.

Зафиксируем натуральные числа $m$ и $n$ такие, что $2 \leqslant m \leqslant n$, и разбиение $\lambda=\left(\lambda_{1}\right.$, $\left.\lambda_{2}, \ldots, \lambda_{m}\right)$ числа $n$ на $m$ частей. Через $\mathbf{S}_{m}$ будем обозначать симметрическую групу на множестве $\{1,2, \ldots, m\}$. Положим

$$
\begin{aligned}
W_{\lambda} & =\left\{u \in F \mid \ell(u)=n, c(u)=\left\{x_{1}, x_{2}, \ldots, x_{m}\right\} \quad \text { и } \operatorname{part}(u)=\lambda\right\} \text { и } \\
\mathbf{S}_{\lambda} & =\left\{\sigma \in \mathbf{S}_{m} \mid \lambda_{i}=\lambda_{\sigma(i)} \text { для всякого } i=1,2, \ldots, m\right\} .
\end{aligned}
$$

Ясно, что $\mathbf{S}_{\lambda}$ - подгруппа в $\mathbf{S}_{m}$. Для всякого слова $u \in W_{\lambda}$ и всякой перестановки $\sigma \in \mathbf{S}_{\lambda}$ обозначим через $\sigma^{*}(u)$ слово, полученное из $u$ заменой каждого вхождения буквы $x_{i}$ в $u$ на букву $x_{\sigma(i)}$ (для всех $\left.i=1,2, \ldots, m\right)$. Из определения групшы $\mathbf{S}_{\lambda}$ непосредственно вытекает, что $\sigma^{*}(u) \in W_{\lambda}$. Очевидно, что множество $W_{\lambda}$ с набором унарных операций $\left\{\sigma^{*} \mid \sigma \in \mathbf{S}_{\lambda}\right\}$ является $\mathbf{S}_{\lambda}$-множеством. 
ЛЕмма 6 [1, теорема 4.1]. Для всякого $\lambda \in \Lambda$ интервал $I_{\lambda}$ антиизоморфен решетке конгруэнций $\mathbf{S}_{\lambda}$-мнохсества $W_{\lambda}$.

Ясно, что если $u \in W_{\lambda}$, а $\sigma$ - неединичная перестановка из $\mathbf{S}_{\lambda}$, то $\sigma^{*}(u) \not \equiv u$ и потому $\operatorname{Stab}_{W_{\lambda}}(u)$ - единичная групша, которую мы будем обозначать через $\mathbf{E}$.

Основным результатом работы является

ТЕОремА 2. Для надкоммутативного многообразия полугрупп $\mathscr{V}$ следующие условия әквивалентны:

а) $\mathscr{V}$ - дистрибутивный әлемент решетки ОС;

б) $\mathscr{V}$ - кодистрибутивный әлемент решетки ОС;

в) $\mathscr{V}$ - стандартный әлемент решетки ОС;

г) $\mathscr{V}$ - костандартный элемент решетки ОС;

д) $\mathscr{V}$ - нейтральный әлемент решетки $\mathbf{O C}$;

е) $\mathscr{V}$ совпадает либо с многообразием всех полугрупп, либо с многообразием вида $\mathscr{C}_{n, m}$, где $m$ и $n$ - натуральные числа такие, что $m \leqslant n u n \neq 1$, либо с многообразием вида $\mathscr{C}_{\lambda}$, где $\lambda \in \Lambda$.

ДокАЗАтЕльство. Импликация е) $\Longrightarrow$ д) легко извлекается из доказательств работы [1], а импликации д) $\Longrightarrow$ в) $\Longrightarrow$ а) и д) $\Longrightarrow$ г) $\Longrightarrow$ б) очевидны. Остается доказать импликации а) $\Longrightarrow$ е) и б) $\Longrightarrow$ е)

Пусть $\mathscr{V}$ - надкоммутативное многообразие полугруш, являющееся дистрибутивным или кодистрибутивным элементом решетки ОС. Вполне инвариантную конгруэнцию на полугруппе $F$, соответствующую многообразию $\mathscr{V}$, обозначим через $\nu$. Ясно, что конгруэнция $\nu$ подкоммутативна, т.е. содержится во вполне инвариантной конгруэнции на $F$, соответствующей многообразию всех коммутативных полугрупп. Обозначим через $\mathbf{S C}$ решетку всех подкоммутативных вполне инвариантных конгруэнций на $F$. Ясно, что эта решетка антиизоморфна решетке ОС. В силу лемм 5 и 6 решетка $\mathbf{S C}$ изоморфна подпрямому произведению решеток вида $\operatorname{Con}\left(W_{\lambda}\right)$, где $\lambda$ пробегает $\Lambda$. При этом из доказательства леммы 5 (данного в [1]) видно, что проекцией на $\operatorname{Con}\left(W_{\lambda}\right)$ образа конгруэнции $\nu$ при изоморфном вложении $\mathbf{S C}$ в $\prod_{\lambda \in \Lambda} \operatorname{Con}\left(W_{\lambda}\right)$ является просто ограничение конгруэнции $\nu$ на $W_{\lambda}$. Обозначим это ограничение через $\nu_{\lambda}$. В силу сказанного выше $\nu_{\lambda}$ является дистрибутивным или кодистрибутивным элементом решетки $\operatorname{Con}\left(W_{\lambda}\right)$. Доказываемые импликации, очевидно, эквивалентны тому, что для всякого $\lambda \in \Lambda$ конгруэнция $\nu_{\lambda}$ является либо универсальным отношением, либо отношением равенства на $W_{\lambda}$. Пусть $\lambda=\left(\lambda_{1}, \lambda_{2}, \ldots, \lambda_{m}\right) \in \Lambda$. Дальнейшие рассмотрения распадаются на два случая.

Случай $1 . \lambda_{1}>1$. В этом случае $W_{\lambda}$ содержит, в частности, слова

$$
u \equiv x_{1}^{\lambda_{1}} x_{2}^{\lambda_{2}} \ldots x_{m}^{\lambda_{m}} \quad \text { и } \quad v \equiv x_{1}^{\lambda_{1}-1} x_{2}^{\lambda_{2}} \ldots x_{m}^{\lambda_{m}} x_{1}
$$

Ясно, что $\sigma^{*}(u) \not \equiv v$ для всякой перестановки $\sigma \in \mathbf{S}_{\lambda}$. Следовательно, $\mathbf{S}_{\lambda}$-множество $W_{\lambda}$ нетранзитивно. Поскольку, как отмечалось перед формулировкой теоремы, стабилизаторы любых двух элементов этого $\mathbf{S}_{\lambda}$-множества совпадают, остается сослаться на теорему 1.

Случай 2. $\lambda_{1}=1$. Ясно, что в этом случае $\lambda_{2}=\cdots=\lambda_{m}=1, \mathbf{S}_{\lambda}=\mathbf{S}_{m}$ и $W_{\lambda}$ есть транзитивное $\mathbf{S}_{m}$-множество. Как хорошо известно (см., например, [6, лемма 4.20]), peшетка конгруэнций транзитивного $G$-множества $A$ изоморфна интервалу $\left[\operatorname{Stab}_{A}(a), G\right]$ решетки подгрупп групшы $G$ (где $a$ - произвольньй элемент из $A$ ). Как обычно, мы будем обозначать решетку подгрупп группы $G$ через $\operatorname{Sub}(G)$. Как отмечалось перед 
формулировкой теоремы, стабилизатор любого элемента $\mathbf{S}_{\lambda}$-множества $W_{\lambda}$ равен $\mathbf{E}$. Следовательно, в рассматриваемом случае $\operatorname{Con}\left(W_{\lambda}\right) \cong \operatorname{Sub}\left(\mathbf{S}_{m}\right)$. Осталось показать, что решетка $\operatorname{Sub}\left(\mathbf{S}_{m}\right)$ не содержит ни дистрибутивных, ни кодистрибутивных элементов, отличных от $\mathbf{S}_{m}$ и $\mathbf{E}$. Решетка $\operatorname{Sub}\left(\mathbf{S}_{2}\right)$ вообще не содержит элементов, отличных от $\mathbf{S}_{2}$ и $\mathbf{E}$. Пусть теперь $m \geqslant 3$. Известно (см., например, [7, теорема 3.4.2]), что если подгрупша $H$ групшы $G$ является дистрибутивным или кодистрибутивным элементом решетки $\operatorname{Sub}(G)$, то $H$ нормальна в $G$ и имеет единственное дополнение в $\operatorname{Sub}(G)$. Как хорошо известно, при $m \neq 4$ единственной нормальной подгруппой в $\operatorname{Sub}\left(\mathbf{S}_{m}\right)$, отличной от $\mathbf{S}_{m}$ и $\mathbf{E}$, является знакопеременная группа $\mathbf{A}_{m}$, а в $\mathbf{S}_{4}$ таких подгрупп две $\mathbf{A}_{4}$ и четверная групша Клейна $\mathbf{V}_{4}$. Ясно, что при любом $m \geqslant 3$ группа $\mathbf{S}_{m}$ содержит более одной транспозиции и подгруппа, порожденная любой транспозицией, является дополнением к $\mathbf{A}_{m}$ в $\operatorname{Sub}\left(\mathbf{S}_{m}\right)$. Группа $\mathbf{V}_{4}$ также содержит более одного дополнения в решетке $\operatorname{Sub}\left(\mathbf{S}_{4}\right)$ - таковыми являются подгрупшы вида

$$
\operatorname{Stab}_{4}(i)=\left\{\sigma \in \mathbf{S}_{4} \mid \sigma(i)=i\right\}
$$

для всех $i=1,2,3,4$. Таким образом, при любом $m$ решетка $\operatorname{Sub}\left(\mathbf{S}_{m}\right)$ не содержит ни дистрибутивных, ни кодистрибутивньх элементов, отличных от $\mathbf{S}_{m}$ и $\mathbf{E}$. Следовательно, и в этом случае $\nu_{\lambda}$ - либо универсальное отношение, либо отношение равенства.

Теорема доказана.

Хорошо известно (см., например, доказательство теоремы III.2.4 в [5]), что если $x-$ нейтральный элемент решетки $L$, то $L$ разлагается в подпрямое произведение главного идеала $(x]$ и главного фильтра $[x)$. Теорема 2 показывает, что подпрямое разложение решетки ОС, найденное в [1], является наиболее дробным в том смысле, что оно использует все нейтральные элементы этой решетки.

Хорошо известно, что решетка ОС континуальна (см., например, [8]). В связи с этим представляет интерес непосредственно вытекающее из теоремы 2.

СлЕДСТВИЕ. Множество всех (ко)дистрибутивных әлементов решетки ОС счетно.

\section{СПИСОК ЦИТИРОВАННОЙ ЛИТЕРАТУРЫ}

[1] Volkov M. V. Young diagrams and the structure of the lattice of overcommutative semigroup varieties // Transformation Semigroups. Proc. Int. Conf. held at the Univ. Essex. Colchester, 1994. P. 99-110.

[2] Vernikov B. M. On congruences of $G$-sets // Comment. Math. Univ. Carol. 1997. V. 38. №3. P. 601-611.

[3] Vernikov B. M. Distributivity, modularity, and related conditions in lattices of overcommutative semigroup varieties // Semigroups with Applications, Including Semigroup Rings. St. Petersburg, 1999. P. 411-439.

[4] Vernikov B. M. Quasiidentities implying modularity and related conditions in lattices of overcommutative semigroup varieties // Semigroup Forum. 1998. V. 57. № 1. P. 142-150.

[5] Гретцер Г. Общая теория решеток. М.: Мир, 1982.

[6] McKenzie R. N., McNulty G.F., Taylor W. F. Algebras. Lattices. Varieties. V. I. Monterey: Wadsworth\&Brooks/Cole, 1987.

[7] Schmidt R. Subgroup Lattices of Groups. Berlin: Walter de Gruyter, 1994.

[8] Evans T. The lattice of semigroup varieties // Semigroup Forum. 1971. V. 2. № 1. P. 1-43. 